CFS of $\geq 6$ (scale $1-9$ with a high score indicating greater frailty)

Results 35 patients received AFM and 10 (29.4\%) were intolerant of treatment at 4 months. Patients without frailty were more likely to be tolerant of treatment than those with frailty (86.4\% versus 33.3\%, $\mathrm{p}=.0074)$ Patients with a BMI in the upper three quartiles of the sample were more likely to be tolerant of treatment than those in the lowest quartile, but this trend did not reach statistical significance $(80.7 \%$ versus $44.4 \%, \mathrm{p}=0.081$ ), and there was no difference in tolerance between patients over 80 years of age and younger patients (77.8\% versus $75.0 \%, \mathrm{p} \geq 0.99$ )

Conclusions Frailty and low BMI may predict treatment intolerance with AFM in IPF, whereas age does not appear to influence this outcome. Clinicians should consider a patient's frailty when considering this therapy. Further analysis of a larger dataset and a prospective study are warranted.

\section{P154 GENDER AND HEIGHT DRIVE VARIATION BETWEEN FORCED VITAL CAPACITY REFERENCE EQUATIONS: IMPLICATIONS FOR IPF TREATMENT}

F Frost, R Peat, S Town, C Brockelsby, L Johns, E Hilal. Liverpool Heart and Chest Hospital, Liverpool, UK

\subsection{6/thoraxjnl-2017-210983.296}

Introduction NICE guidance mandates that anti-fibrotic therapy for interstitial pulmonary fibrosis (IPF) is only recommended as on option for people with a forced vital capacity (FVC) between 50\%-80\% predicted. The guidance recognises different reference values are used across the UK to calculate predicted FVC but does not specify which should be utilised. We were interested to see how different formulas impacted on eligibility for these treatments.

Methods We reviewed all patients with a diagnosis of IPF or possible IPF attending our ILD clinic for the time period 2016-2017. Baseline demographics were recorded and $\%$ predicted FVC (ppFVC) was calculated using both the European Society for Coal and Steel (ESCS) and Global Lung Initiative 2012 (GLI) formulas.

Results We identified 97 patients from our database and complete data was available for 96 (median age [range] 71.2 years (42-89), 62.5\% male, median FVC 2.39 L). Overall the ESCS formula resulted in a higher ppFVC compared to GLI $(+6.2 \%$ FVC,$p<0.001)$. There was considerably less difference between the formulas in males than females $(+3.7 \%$ vs. $+11.6 \%, \mathrm{p}<0.0001)$. We observed a strong inverse correlation between variation in ppFVC and height (Rho -0.7, $\mathrm{p}<0.0001)$. No relationship was observed between age and variation. For the 24 patients with GLI ppFVC within 10\% of the upper and lower thresholds for treatment, 15 (62.5\%) would have their eligibility for anti-fibrotic treatments changed by use of the ESCS formula.

Conclusions Females and those with shorter height saw the greatest variation between the two formulas. A significant proportion of patients with borderline eligibility for anti-fibrotic treatments have their status changed by the use of a different formula. Clinicians must be aware of their local reference values and how this may affect patients' eligibility for IPF treatment.

\section{P155 USE OF MYCOPHENOLATE MOFETIL AND AZATHIOPRINE IN PATIENTS WITH CHRONIC HYPERSENSITIVITY PNEUMONITIS}

CA Fiddler, N Simler, M Thillai, H Parfrey. Papworth Hospital NHS Foundation Trust, Papworth Everard, UK

\subsection{6/thoraxjnl-2017-210983.297}

Background The optimal pharmacological management of chronic hypersensitivity pneumonitis (cHP) is unknown. Corticosteroids are often used as first line therapy but can be associated with side effects. There is a paucity of data examining the roln DLCO but not FVC (Morisset J et al. Chest. 2017). We aimed to determine the effece of steroid-sparing agents in cHP. A recent retrospective study demonstrated that treatment with either mycophenolate mofetil (MMF) or azathioprine (AZA) was associated with improvements it of MMF and AZA on lung function and prednisolone dose in cHP patients. Methods Patients initiated on either MMF or AZA following a MDT diagnosis of cHP were retrospectively identified from the ILD service Papworth Hospital, Cambridge. Changes in lung function in the 9-12 months before and after treatment initiation were analysed. Daily prednisolone dose at initiation and 9-12 months treatment was recorded.

Results Twenty eight patients were identified between 2008 and 2016; 20 were treated with MMF (1-2 g daily) and 8 with AZA (25-150 mg daily). The mean age at drug initiation was $59.6 \pm 1.7$ years and $61 \%$ were female. The mean duration from diagnosis to commencing MMF or AZA was 30.9 \pm 5.5 months. Twenty patients remained on either drug at 912 months and were include in the effectiveness analysis (FVC and TLCO data were available for 20 and 13 patients respectively). Five patients discontinued treatment due to drug side effects. Treatment with either MMF or AZA resulted in a significant reduction in prednisolone dose from $16.1 \pm 2.1 \mathrm{mg}$ to $8.0 \pm 0.8 \mathrm{mg}(\mathrm{p}<0.001)$. MMF or AZA treatment for $9-12$ months was associated with a significant improvement in TLCO $\quad(-0.62 \pm 0.3 \mathrm{vs}+0.32 \pm 0.17 \mathrm{mmol} / \mathrm{kPa} / \mathrm{min}, \quad \mathrm{p}<0.05)$. Although treatment reduced rate of FVC decline $(-100 \pm 65$ vs $-30 \pm 66 \mathrm{mls})$, it was not significant $(p=0.4)$.

Conclusions In our cohort of cHP, treatment with either MMF or AZA was associated with an improvement in TLCO consistent with findings of a previous retrospective study. Moreover, the addition of MMF or AZA enabled a significant reduction in prednisolone dose.

\section{P156 MAINTAINING PATIENTS WITH IDIOPATHIC PULMONARY FIBROSIS (IPF) ON ANTIFIBROTIC THERAPY; THE NURSES' CHALLENGE}

GA Burge, H Alldrick, E Briggs, K Neighbour, PS Burge, GI Walters. Heart of England NHS Foundation Trust, Birmingham, UK

\subsection{6/thoraxjnl-2017-210983.298}

Pirfenidone and nintedanib are the first licensed drugs for IPF. Both reduce the rate of disease progression but have significant intolerability issues limiting long-term use. This study aims to identify areas where changes in practice might improve outcomes. We compared baseline characteristics of disease severity with the reasons for stopping treatment and the proportion stopping treatment within the first 4 weeks, 
for all patients from our centre up to December 2016, including those receiving treatment on a named patient basis. Data was available for $150 / 153$ patients started on pirfenidone and $56 / 57$ on nintedanib. Table 1 compares patients who were dispensed treatment but never took it, patients who stopped after the first prescription, and those continuing treatment. 8 patients consented to treatment but never took the drug; they were older and had less advanced disease. 39 patients stopped treatment after the first prescription; they had slightly more advanced disease, and had a longer interval between drug delivery and the first nurse (60.7 SD 57 days vs. 45.4 SD 30; $(\mathrm{p}=0.032)$, and more often had a shared care arrangement, but did not have a lower BMI. Photosensitivity for pirfenidone and diarrhoea for nintedanib were usually managed by treatment and were uncommon reasons for stopping antifibrotic therapy, which was more often related to upper GI intolerance and disease progression.

Conclusion Patients stopping antifibrotic treatment early cannot be identified from baseline data but can be reduced by intensive nursing support. Prescribing centre based ILD-CNS's should be responsible for early treatment tolerability and aim to see patients monthly until established on treatment. Uncertain delays between prescription and drug delivery make this more difficult.

\begin{tabular}{|c|c|c|c|}
\hline & \multicolumn{3}{|c|}{ Prescription dispensed } \\
\hline & $\begin{array}{l}\text { Once and never } \\
\text { taken }\end{array}$ & $\begin{array}{l}\text { Once and not } \\
\text { repeated }\end{array}$ & continued \\
\hline Number & 8 & 39 & 159 \\
\hline FVC\% predicted (SD) & $89.5(27)$ & $69.3(16)$ & $74.8(14)$ ** \\
\hline Composite physiology & $36.4(16)$ & 56.0() & $48.9(10)$ \\
\hline index & & & $* * *$ \\
\hline Shared care\% & & 38 & 20 ** \\
\hline Age & $78.0(6)$ & $72.5(7)$ & $70.4(9)$ * \\
\hline BMI & $30.0(2)$ & $28.5(6)$ & $29.2(5)$ \\
\hline
\end{tabular}

\section{P157 CAN BASELINE PHYSIOLOGICAL TESTS HELP PREDICT THE OUTCOME OF HYPOXIC CHALLENGE TESTING (HCT) IN INTERSTITIAL LUNG DISEASE (ILD)?}

${ }^{1} \mathrm{SL}$ Barratt, ${ }^{2} \mathrm{~J}$ Shaw, ${ }^{1} \mathrm{R}$ Jones, ${ }^{1} \mathrm{H}$ Adamali, ${ }^{3} \mathrm{C}$ Cliff, ${ }^{2} \mathrm{~N}$ Clayton, ${ }^{3} \mathrm{~N}$ Mustfa, ${ }^{3} \mathrm{H}$ Stone, ${ }^{2} \mathrm{~N}$ Chaudhuri. ${ }^{1}$ Bristol interstitial Lung Disease Service, Bristol, UK; ${ }^{2}$ University Hospital of South Manchester, Manchester, UK; ${ }^{3}$ Royal Stoke University Hospital, Stoke, UK

\subsection{6/thoraxjnl-2017-210983.299}

Introduction The British Thoracic Society guidelines suggest that pre-flight risk assessment should be considered in all individuals with Interstitial Lung Disease (ILD) intending to undertake commercial air travel. Hypoxic challenge testing (HCT) can be used to aid decisions about the need for inflight oxygen but there is a lack of evidence as to which patient variables might predict the outcome of HCT to guide referral for assessment.

Objective To investigate variables that might predict a hypoxaemic response to HCT in patients with ILD.

Methods A multi-centre retrospective analysis of all ILD patients attending for HCT at three tertiary care ILD referral centres between January 2010 and March 2017 was undertaken. The outcome of HCT was correlated to baseline demographic data, oxygen saturations (SpO2), capillary ear lobe $\mathrm{PaO} 2$, pulmonary function testing, 6MWT and GAP index, performed within 6 months of the HCT. Groups were compared using unpaired t-test with Welch's correction, unless otherwise stated $\quad(\mathrm{p}<0.05$ was considered statistically significant).

Results A total of 106 ILD patients (61 of whom (58\%) had IPF) underwent HCT. Of these, 54 (51\%) patients (of whom $30(49 \%)$ had IPF) failed HCT and were recommended supplemental in-flight oxygen. ILD patients who failed HCT had significantly lower resting SpO2, FEV1, FVC and TLCO $\%$ predicted, but higher GAP index (Table 1). In addition to these variables, the IPF subgroup failing HCT also had significantly lower minimum SpO2 during 6MWT.

Conclusions To our knowledge this is the largest retrospective study exploring predictors of HCT outcomes in ILD. Several baseline physiological parameters are significantly different between those ILD patients requiring in-flight oxygen based on HCT, and those who do not, including in a well-defined subgroup of IPF patients. Work is underway to establish a risk model to guide clinician decisions regarding the need for HCT in ILD.

\section{P158 PULMONARY VASCULAR DISEASE MARKERS PREDICT DEATH IN INTERSTITIAL LUNG DISEASE PATIENTS PROVEN NOT TO HAVE PULMONARY HYPERTENSION AT RIGHT HEART CATHETER}

${ }^{1}$ SRB Bax, ${ }^{1} \mathrm{C}$ Breedy, ${ }^{1} \mathrm{~K}$ Dimopoulos, ${ }^{1} \mathrm{~A}$ Kempny, ${ }^{1} \mathrm{~A}$ Devaraj, ${ }^{2} \mathrm{~S}$ Walsh, ${ }^{1} \mathrm{~J}$ Joseph, ${ }^{3} \mathrm{~S}$ Nair, ${ }^{1} \mathrm{M}$ Kokosi, ${ }^{4} \mathrm{G}$ Kier, ${ }^{1} \mathrm{C}$ Harries, ${ }^{1} \mathrm{~V}$ Kouranos, ${ }^{1} \mathrm{C}$ McCabe, ${ }^{1} \mathrm{~W}$ Li, ${ }^{5} \mathrm{M}$ Wilde, ${ }^{1} \mathrm{AU}$ Wells, ${ }^{1}$ LC Price*, 'SJ Wort*. ${ }^{1}$ Royal Brompton Hospital, London, UK; ${ }^{2}$ Kings College Hospital Foundation Trust, London, UK; ${ }^{3}$ Guy's and St Thomas' NHS Foundation Trust, London, UK; ${ }^{4}$ PrincessAlexandra Hospital, Brisbane, Australia; ${ }^{5}$ Surrey and Sussex NHS Trust, London, UK; *Joint final author

\subsection{6/thoraxjnl-2017-210983.300}

Introduction Patients with interstitial lung disease (ILD) often have signs of pulmonary hypertension $(\mathrm{PH})$ when assessed non-invasively. The presence of pulmonary hypertension $(\mathrm{PH})$ at right heart catheter (RHC) is a negative prognostic factor; however, the impact of elevated pulmonary vascular biomarkers in the absence of $\mathrm{PH}$ is poorly understood. We hypothesised that pulmonary vascular disease biomarkers would predict mortality in patients without PH at RHC.

Methods Demographics, ILD subtype, PFTs, echocardiogram, and CTs were reviewed in consecutive patients undergoing right heart catheterisation (RHC) for suspected ILD-PH. Patients with a mean pulmonary arterial pressure (mPAP) $<25 \mathrm{mmHg}$ at RHC were studied. Predictors of prognosis were evaluated in their ability to predict mortality using Cox proportional hazard analysis. 
Abstract P157 Table 1 Physiological variables of Interstitial Lung Disease patients referred for hypoxic challenge testing (HCT). All statistical analyses performed using unpaired t-test with Welch's Correction, except as indicated by ${ }^{\wedge}$ where Fisher's exact test was used. ( ${ }^{*} p<0.05$, ${ }^{* *} p<0.01,{ }^{* * *} p<0.001,{ }^{* * * *} p<0.0001, n=$ number of patients, S.D.=standard deviation)

\begin{tabular}{|c|c|c|c|c|c|c|c|c|c|c|}
\hline & \multicolumn{5}{|c|}{ Interstitial Lung Disease } & \multicolumn{5}{|c|}{ Idiopathic Pulmonary Fibrosis } \\
\hline & \multicolumn{2}{|l|}{ Failed HCT } & \multicolumn{2}{|l|}{ Passed HCT } & \multirow[t]{2}{*}{$P$ value } & \multicolumn{2}{|l|}{ Failed HCT } & \multicolumn{2}{|l|}{ Passed HCT } & \multirow[t]{2}{*}{$P$ value } \\
\hline & Mean (S.D) & $\mathrm{n}$ & Mean (S.D) & $\mathbf{n}$ & & Mean (S.D.) & $\mathbf{n}$ & Mean (S.D.) & $\mathbf{n}$ & \\
\hline Gender & $36 \mathrm{M}: 18 \mathrm{~F}$ & 54 & $38 \mathrm{M}: 14 \mathrm{~F}$ & 52 & $0.5293^{\wedge}$ & $24 \mathrm{M}: 6 \mathrm{~F}$ & 30 & $24 \mathrm{M}: 7 \mathrm{~F}$ & 31 & $1.0000^{\wedge}$ \\
\hline Age (years) & $69.44(8.99)$ & 54 & $68.80(8.54)$ & 52 & 0.7091 & $70.80(7.57)$ & 30 & $72.48(6.76)$ & 31 & 0.3638 \\
\hline FEV1 (litres) & $1.90(0.62)$ & 53 & $2.20(0.57)$ & 52 & $0.0092^{* *}$ & $2.00(0.62)$ & 30 & $2.25(0.56)$ & 31 & 0.1072 \\
\hline FEV1\% predicted & $73.83(20.36)$ & 53 & $81.96(16.93)$ & 52 & $0.0282^{*}$ & $71.97(13.62)$ & 30 & $85.65(16.08)$ & 31 & $0.0007^{* * *}$ \\
\hline FVC (litres) & $2.31(0.78)$ & 54 & $2.72(0.73)$ & 52 & $0.0059 * *$ & $2.40(0.73)$ & 30 & $2.72(0.72)$ & 31 & 0.0990 \\
\hline FVC\% predicted & $72.20(22.53)$ & 54 & $80.14(17.33)$ & 52 & $0.0429^{*}$ & $68.53(13.45)$ & 30 & $81.23(16.63)$ & 31 & $0.0018^{* *}$ \\
\hline FEV1:FVC (\%) & $81.42(6.78)$ & 53 & $80.94(7.25)$ & 52 & 0.7309 & $82.00(13.62)$ & 30 & $82.41(7.41)$ & 31 & 0.8120 \\
\hline TLCO & $3.21(0.89)$ & 46 & $4.35(1.45)$ & 47 & $<0.0001 * * * *$ & $3.29(0.93)$ & 24 & $4.03(1.13)$ & 26 & $0.0143^{*}$ \\
\hline TLCO $\%$ predicted & $41.04(10.77)$ & 46 & $52.64(14.47)$ & 47 & $<0.0001 * * * *$ & $40.08(8.66)$ & 24 & $50.50(14.14)$ & 26 & $0.0029^{* *}$ \\
\hline KCO & $1.04(0.29)$ & 45 & $1.17(0.35)$ & 47 & 0.0608 & $1.02(0.25)$ & 24 & $1.06(0.27)$ & 26 & 0.5526 \\
\hline KCO $\%$ predicted & $79.49(22.48)$ & 45 & $86.96(23.48)$ & 47 & 0.1226 & $81.33(22.00)$ & 24 & 83.92 (19.14) & 26 & 0.6603 \\
\hline Baseline $\mathrm{PO}_{2}(\mathrm{kPa})$ & $9.00(1.06)$ & 51 & $9.67(1.64)$ & 50 & $0.0175^{*}$ & $9.00(1.01)$ & 29 & $9.24(1.66)$ & 31 & 0.5058 \\
\hline Resting $\mathrm{SpO}_{2}(\%)$ & $94.14(3.37)$ & 53 & $95.43(2.03)$ & 52 & $0.0198^{*}$ & $94.13(3.06)$ & 30 & $95.53(1.88)$ & 31 & $0.0377^{\star}$ \\
\hline GAP index & $4.31(1.44)$ & 51 & $3.42(1.39)$ & 50 & $0.0019^{* *}$ & $4.73(1.11)$ & 30 & $3.79(1.05)$ & 29 & $0.0015^{* *}$ \\
\hline 6MWT & 308.42 & 33 & 365.90 & 39 & $0.0236^{*}$ & 316.45 & 20 & 349.39 & 23 & 0.3526 \\
\hline -actual distance(m) & $(120.08)$ & 32 & $(81.90)$ & 38 & $0.0493^{*}$ & (134.89) & 19 & (84.29) & 22 & 0.1745 \\
\hline$-\%$ theoretical distance & $64.94(23.93)$ & 33 & $75.29(18.15)$ & 39 & 0.0780 & $64.00(26.37)$ & 20 & $74.14(19.17)$ & 23 & $0.0043^{* *}$ \\
\hline (\%) & $85.46(6.06)$ & 17 & $87.77(4.65)$ & 30 & 0.8782 & $84.20(6.22)$ & 6 & $89.04(3.37)$ & 13 & 0.0614 \\
\hline $\begin{array}{l}\text {-minimum } \mathrm{SpO}_{2}(\%) \\
\text {-maximum heart rate } \\
(\mathrm{bpm})\end{array}$ & $112.12(11.73)$ & & $\begin{array}{l}111.47 \\
(17.10)\end{array}$ & & & $111.00(4.94)$ & & $\begin{array}{l}102.31 \\
(13.82)\end{array}$ & & \\
\hline
\end{tabular}

Results Between 2005 and 2015, 68 patients (47\% male) were evaluated that did not subsequently have PH (mPAP at RHC $19 \pm 4 \mathrm{mmHg}$; Pulmonary vascular resistance (PVR) $2.5 \pm 1.4$ Wood units). On CT scanning main pulmonary artery diameter (MPAdiam) was $29.9 \pm 5 \mathrm{~mm}$ and main pulmonary artery to aorta ratio (MPAdiam:aa) was $0.97 \pm 0.1$. Median brain natriuretic peptide (BNP) was 44[29-72] (normal <20 ng.L) and predicted right ventricular systolic pressure (RVSP) at echocardiogram was $48 \pm 13 \mathrm{mmHg}$. Forced vital capacity (FVC) was $62 \% \pm 22 \%$ predicted. PVR as a continuous variable predicted mortality (hazard ratio $(\mathrm{HR}): 1.35, \mathrm{p}=0.02$ ) per unit increase. A MPAdiam $\geq 32 \mathrm{~mm}$ was associated with mortality (hazard ratio $(\mathrm{HR}): 3.02, \mathrm{p}=0.001)$ as was MPAdiam:aa ratio $\geq 0.9$ (HR:4.05, p=0.001). BNP $\geq 40$ ng.L (HR:2.47, $p=0.02$ ) and a RVSP $\geq 40 \mathrm{mmHg}$ (HR:2.74, $\mathrm{p}=0.02$ ) also predicted mortality. MPAdiam and PVR (expressed as a continuous variable) remained independent predictors of mortality after adjusting for ILD diagnosis, forced vital capacity (\% predicted) and age at RHC.

Conclusion Even in the absence of $\mathrm{PH}$ at RHC, elevated pulmonary vascular biomarkers are useful in risk stratification of patients suspected of having ILD associated $\mathrm{PH}$.

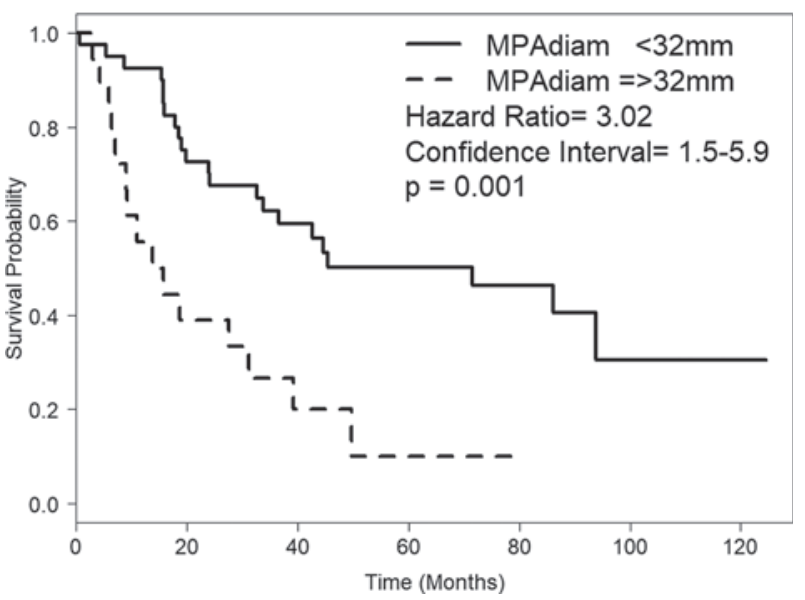

Abstract P158 Figure 1 Main pulmonary artery diameter. 